\title{
Laparoscopic distal gastrectomy with intracorporeal handsewn Billroth-I anastomosis (ICHSA)
}

\author{
Katsuichi Matsuo $\cdot$ Hideo Shimura $\cdot$ Shinnosuke Tanaka $\cdot$ Masahiko Nakano $\cdot$ \\ Tatsuya Hashimoto $\cdot$ Daibou Kojima $\cdot$ Yuichi Yamashita $\cdot$ Ken Inoue • \\ Hiroshi Satoh $\cdot$ Asao Inoue
}

Received: 20 August 2011/Accepted: 25 November 2011/Published online: 6 September 2012

(C) The Author(s) 2012. This article is published with open access at Springerlink.com

\begin{abstract}
Background The number of cases of laparoscopic surgery has been increasing. Lymph node dissection has been standardized, and the enlarged view provided by laparoscopes allows for the procedure to be performed successfully entirely within the abdominal cavity, but many cases of reconstruction using the Billroth-I method are performed under direct vision through a small incision. In this study, by placing an anchor thread on a suture line on the lesser curvature of the stomach, we simplified the procedure for handsewn anastomosis and safely performed gastroduodenal anastomosis at low cost to obtain good results.

Methods From January 2009 to December 2010, we performed handsewn gastroduodenal anastomosis in 18 cases. After performing lymph node dissection, the duodenum and the stomach were separated using an automatic stapling device. Anchor sutures were placed on the suture line of the lesser curvature of the stomach. First, the seromuscular layer of the stomach and the seromuscular layer of the duodenum were sutured by performing
\end{abstract}

Presented at the 19th EAES Congress, June 15-18, 2011, Torino, Italy.

Electronic supplementary material The online version of this article (doi:10.1007/s00464-011-2107-1) contains supplementary material, which is available to authorized users.

K. Matsuo $\cdot$ H. Shimura $(\bowtie) \cdot$ S. Tanaka $\cdot$ M. Nakano ·

T. Hashimoto · D. Kojima · Y. Yamashita · K. Inoue ·

H. Satoh · A. Inoue

Department of Gastrointestinal Surgery, Fukuoka University

School of Medicine, 7-45-1 Nanakuma, Jonan-ku,

Fukuoka 810-0180, Japan

e-mail: shimura@fukuoka-u.ac.jp

K. Matsuo

e-mail: katsu-m@db3.so-net.ne.jp interrupted suturing using an extracorporeal knot-tying method. With the stomach and the duodenum in a fixed state, the anastomosis area was opened. The thread of the anchor suture was pulled toward the abdominal wall, and then all layers of the stomach and the duodenum at the posterior wall were continuously sutured. Similarly, for the anterior wall, all layers were continuously sutured from the lesser curvature toward the greater curvature.

Results We performed this anastomotic procedure in 18 patients with early gastric carcinoma. The mean time required for the anastomosis was $64.6 \pm 17.1 \mathrm{~min}$, and the estimated blood loss was $53.1 \pm 91 \mathrm{~g}$. All operations were curative, and the mean number of retrieved lymph node was $27.1 \pm 10.8$. A nasogastric tube was removed on the first or second day. An upper gastrointestinal series performed on postoperative days 5-6 showed no anastomotic leakage and normal transit. Oral intake was started on days 6-7. Postoperative complications included one case of a ruptured suture, but this was resolved through a conservative approach. There was no mortality. Postoperative endoscopy revealed that the anastomosis area was extremely soft, and no abnormalities were observed. Moreover, the only costs related to the anastomosis were for the thread and needles, and although more time was required compared with mechanical anastomosis, the cost was extremely low.

Conclusions We performed gastroduodenal anastomosis under a total laparoscopic approach by handsewn. This method is economical, because it does not require the use of machinery for anastomosis, and the duodenal stump is short. We believe that this method, which can be performed in a similar manner even for obese patients, can be used as a standard method of anastomosis.

Keywords Intracorporeal handsewn anastomosis . Gastric cancer · Billroth-I anastomosis 
A Billroth-I gastrectomy was performed and followed by a handsewn gastroduodenostomy, so Billroth-I anastomosis with handsewn sutures has been widely accepted until now. However, in recent years, laparoscope-assisted surgery for early cancer has been increasing, and in a laparoscopic distal gastrectomy, the method in which a small incision is made at the time of reconstruction is common, which is referred to as laparoscope-assisted surgery [1, 2]. There are various methods of anastomosis for performing mechanical anastomosis entirely within the abdominal cavity, and each method requires at least three or six automatic suture devices [3-5]. Handsewn anastomosis under a total laparoscopic approach was first reported by Takiguchi et al. [6], but due to the large amount of time required for anastomosis and the complexity of the procedure, it is currently not widely performed. In this study, by improving the procedure and placing an anchor thread on a suture line on the lesser curvature of the stomach, we were able to perform handsewn anastomosis easily and entirely within the abdominal cavity and we safely performed handsewn gastroduodenal anastomosis at a low cost to obtain good results. We herein report our findings.

\section{Patients and methods}

From January 2009 to December 2010, we performed handsewn gastroduodenal anastomosis in 18 cases $(10$ males and 8 females) at our facility. Before surgery, we prepared images of the angioarchitecture of the celiac artery by conducted gastric endoscopic examinations, gastric fluoroscopy, and three-dimensional CT examinations.

The patient was fixed in a supine position with her legs spread and her head elevated. A trocar was inserted into the upper part of the umbilicus using an open method to observe the inside of the abdominal cavity using the pneumoperitoneum method. Six ports were inserted into the abdomen.
We determined the position of the port by considering the fact that the gastroduodenum would be sewn by hand (Fig. 1). First, we targeted the gastroduodenum and performed lymph node dissection inside the abdominal cavity. The duodenal bulb and the gastric corpus were separated by using an endoscopic stapling device. Samples were extracted using a large plastic bag after extending the umbilical wound to $3 \mathrm{~cm}$ and fixing a drape for the wound margin.

For reconstruction, the stump of the greater curvature of the remaining stomach and the duodenal stump were anastomosed entirely through handsewn suturing. At the time of the anastomosis, the scope was moved to the $12 \mathrm{~mm}$ trocar in the lower right abdomen in consideration of hand-eye coordination (Fig. 2). Intracorporeal handsewn anastomosis was performed from the right side using two sets of forceps. First, a 3-0 absorbing thread $(120 \mathrm{~cm})$ was placed in the lesser curvature as a supporting thread. Next, a seromyotomy of the stomach was performed (Fig. 3). For the posterior wall, the gastric seromuscular layer and the duodenal seromuscular layer were sewn with four stitches using 3-0 absorbent thread $(120 \mathrm{~cm})$ using an extracorporeal ligation method to repair the stomach and the duodenum (Fig. 4). Next, the duodenal stump and the remaining gastric stump were resected by using an ultrasonic activated device, and each lumen was opened by pulling the supporting thread in the lesser curvature (Fig. 5). Regarding anastomosis of the posterior wall, all of the gastric layers and all of the duodenal layers were consecutively sutured from the lesser curvature side in the direction of the greater curvature using 3-0 coated polyglactin $(17 \mathrm{~cm})$ by continuous suture (Fig. 6). Similarly, for the anterior wall, all of the gastric layers and all of the duodenal layers were consecutively sutured from the lesser curvature side using 3-0 coated polyglactin $(17 \mathrm{~cm})$. The intraabdominal Billroth-I anastomosis is then accomplished (Fig. 7). All of the threads were fixed by performing intracorporeal or extracorporeal knot tying. The intra-abdominal Billroth-I
Fig. 1 Trocar placement for totally laparoscopic distal gastrectomy. Sizes and locations of six abdominal trocar ports used for laparoscopic operation
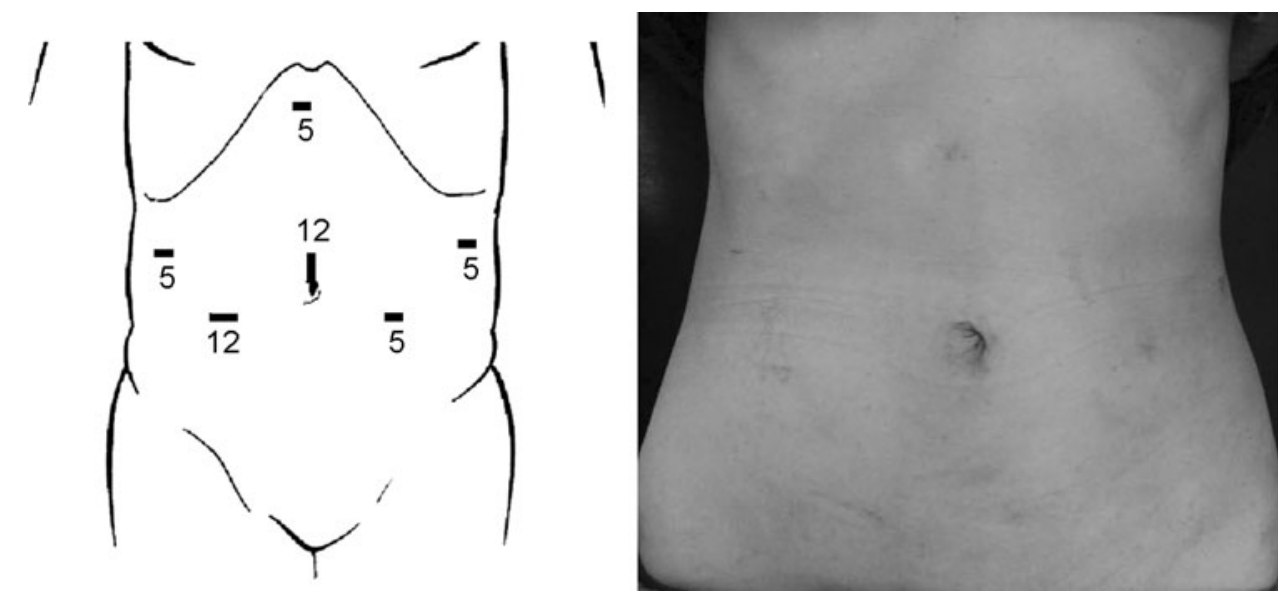
Fig. 2 Coaxial set up to the laparoscopic ports for suturing

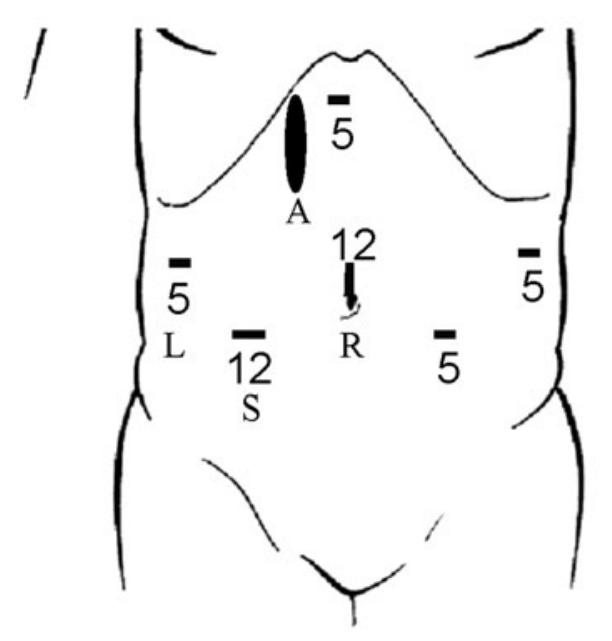

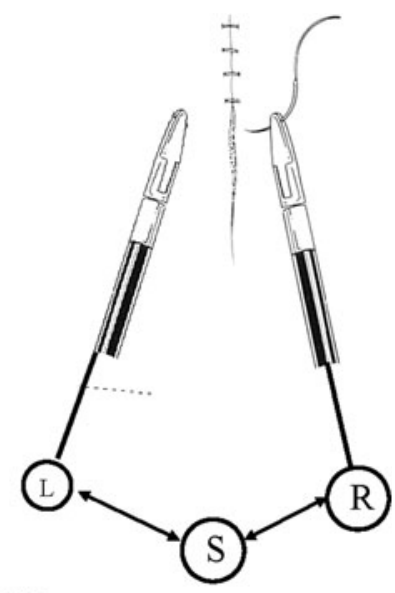

A: Anastomotic area

S: Scope

R: Right hand

L: Left hand

Fig. 3 3-0 absorbing thread $(120 \mathrm{~cm})$ was placed in the lesser curvature as a supporting thread. A seromyotomy of the stomach was performed at the posterior wall

Fig. 4 The posterior seromuscular wall is approximated by using 3-0 absorbing thread in a interrupted fashion
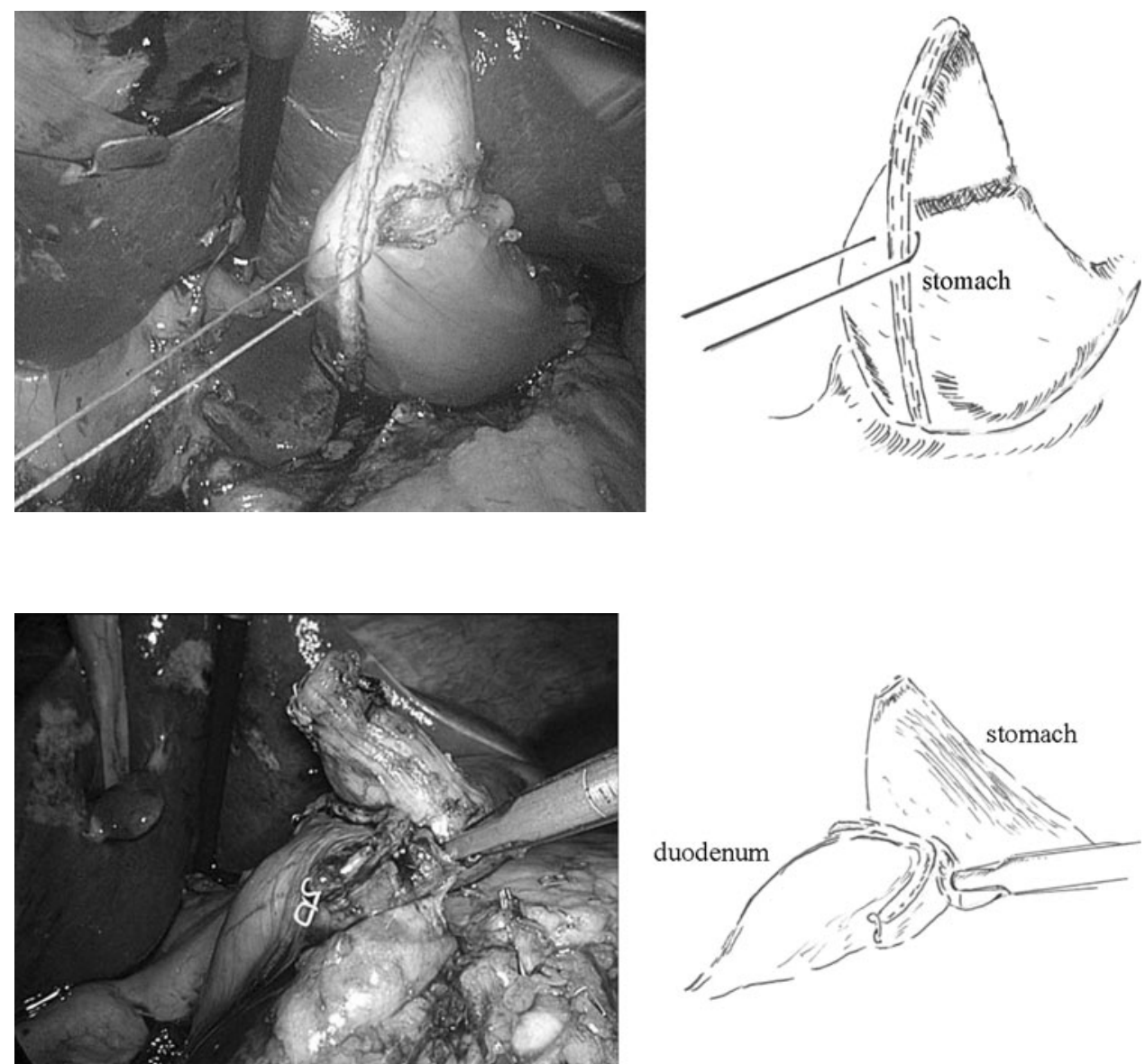

anastomosis is then accomplished. The field of vision in the tangential direction can be improved by pulling the lesser curvature of the stomach toward the abdominal wall with anchor suture threads.
Anastomosis was performed for two layers of the posterior wall and one layer of the anterior wall. After lavaging the abdominal cavity, a closed-end drain was inserted in the posterior surface of the anastomosis area. 
Fig. 5 The duodenal stump and the remaining gastric stump were resected by using an ultrasonic activated device, and each lumen was opened by pulling the supporting thread in the lesser curvature
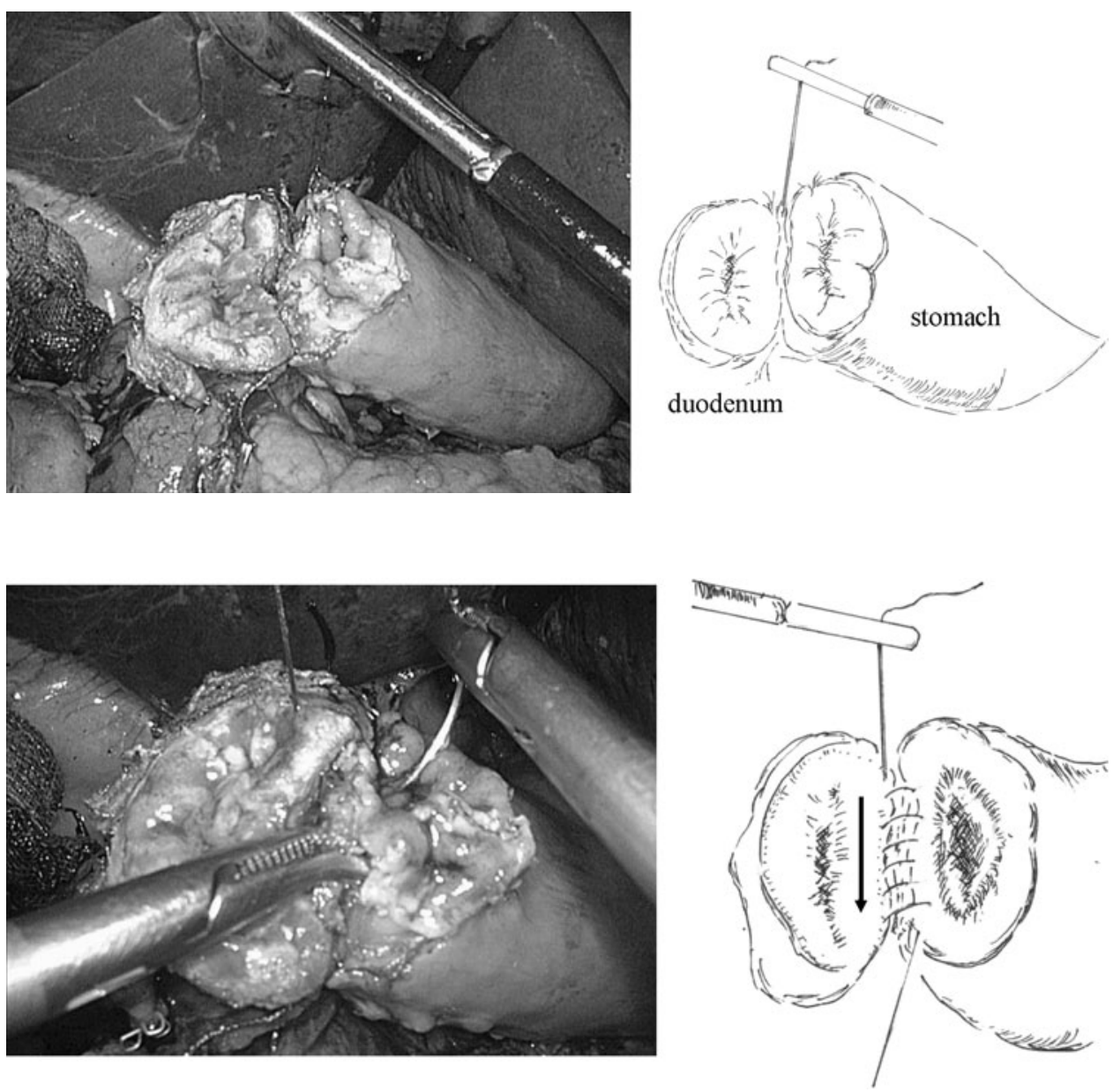

Fig. 6 Running suture of posterior wall by using 3-0 absorbing thread $(17 \mathrm{~cm})$. The lesser curvature to the greater curvature. It is started from the lesser curvature to greater curvature

Fig. 7 Running suture of anterior wall by using 3-0 absorbing thread $(17 \mathrm{~cm})$ was completed. Whole-layer approximation is completed with continuous suture by using handsewn anastomosis technique. The field of vision in the tangential direction can be improved by pulling the lesser curvature of the stomach toward the abdominal wall with anchor suture threads
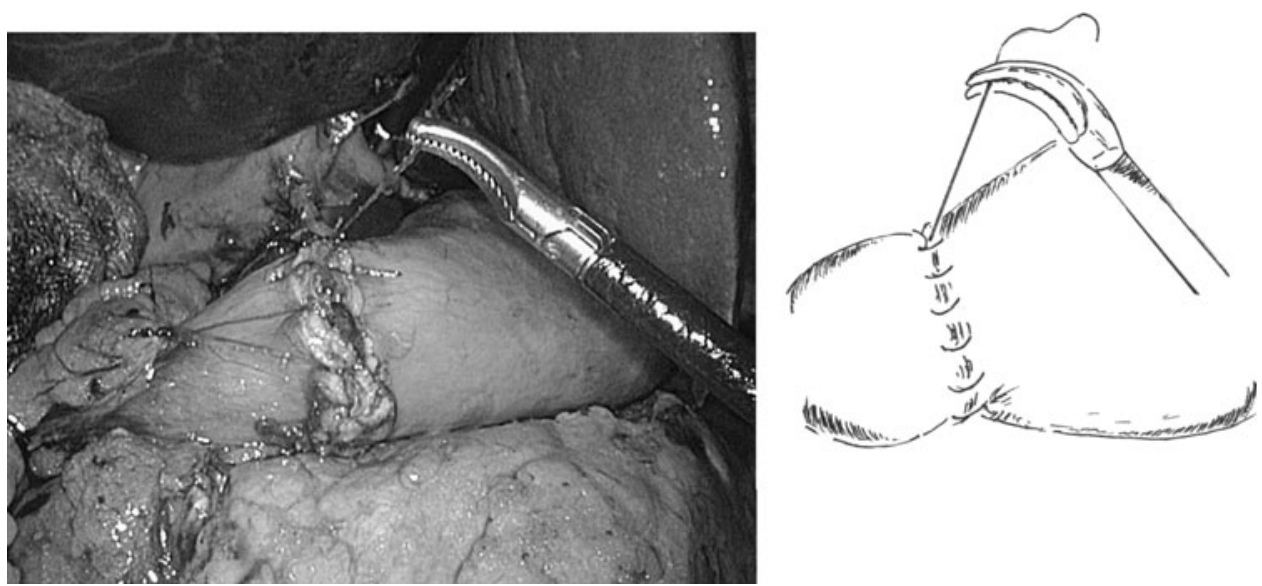

\section{Results}

We performed this anastomotic procedure in 18 patients with early gastric carcinoma. The mean time required for the anastomosis was $64.6 \pm 17.2$ (range, 35-97) minutes, and the estimated blood loss was $53.1 \pm 91 \mathrm{~g}$. All operations were curative, and the mean number of retrieved lymph nodes was $27.1 \pm 10.9$ (range, 11-52; Fig. 8). A nasogastric tube was removed on the first or second day. An upper gastrointestinal series per formed on the postoperative days 5-6 showed no anastomotic leakage and normal transit (Fig. 9). Oral intake was started on days 6-7. One patient developed anastomotic leakage and was treated conservatively. With a mean follow-up of 9.1 (range, 1-19) months, no one suffered from symptoms indicative of anastomotic stenosis, bile reflex, or dumping. There was no mortality. 


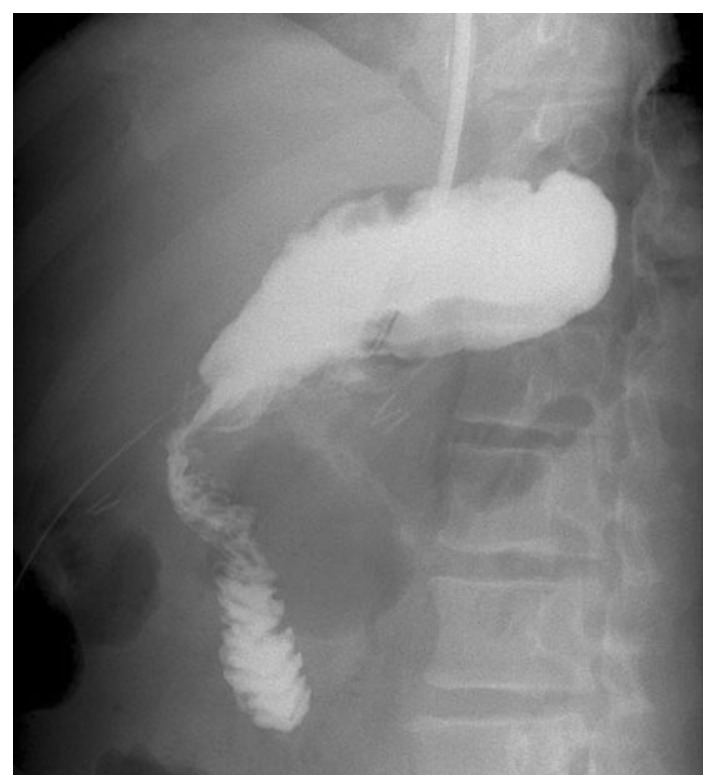

Fig. 8 Fluorography on postoperative demonstrating smooth passage of hydrosoluble contrast without leakage or stenosis of the anastomosis

Postoperative endoscopy revealed that the anastomosis area was extremely soft, and no abnormalities were observed (Fig. 9). Moreover, the only costs related to the anastomosis were for the thread and needles, and although more time was required compared to mechanical anastomosis, the cost was extremely low. The mean duration of hospitalization after surgery was 21.7 (range, 11-37) days.

The running curve of the anastomosis time is shown. Although there is some divergence in the anastomosis time, the linear approximate curve declined (Fig. 10).

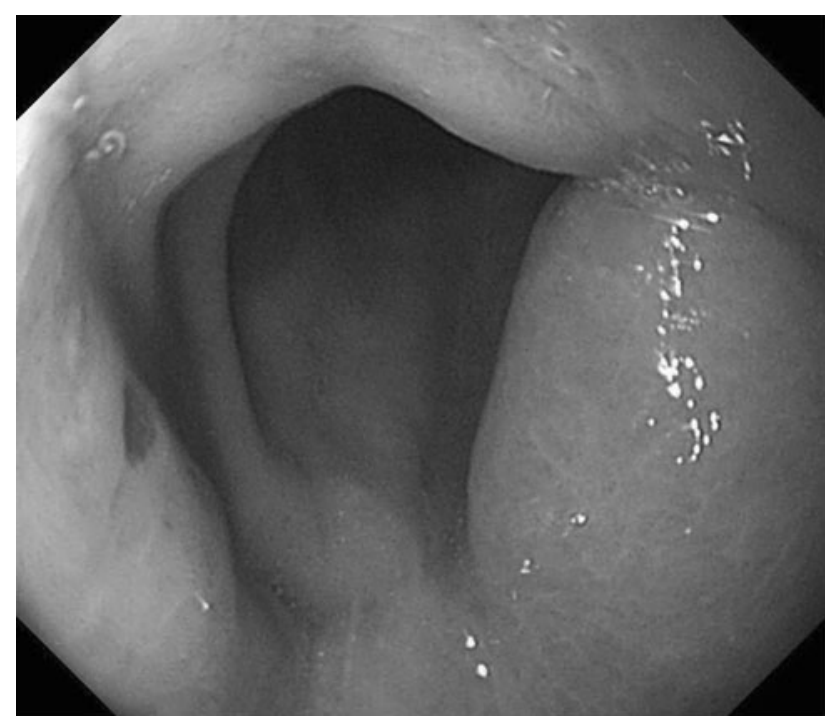

Fig. 9 Endoscopic view of the anastomosis 3 months after the operation

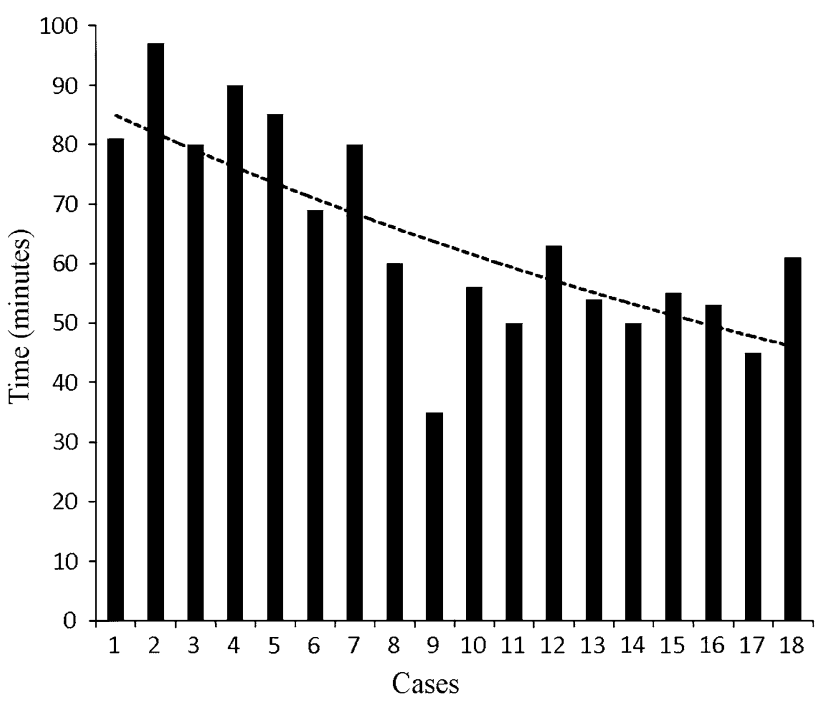

Fig. 10 Learning curve of the anastomotic suture time. Dotted line linear approximate curve

\section{Discussion}

Laparoscopic surgery is understood as a very minimally invasive procedure in the various fields of surgery. Total laparoscopy is close to being the established procedure for laparoscopic lymph node dissection, particularly for treating gastric cancer. However, it is difficult to say that methods for reconstruction after resection have reached a level similar to laparotomy. Complications of LADG, including hemorrhage, intraperitoneal abscess, pancreatic fistula, ileus, respiratory complications, and wound infection, account for up to $1 \%$, whereas complications regarding reconstruction for ruptured sutures and stenosis of the anastomotic part, etc., account for 1 to $6 \%$ [7-12]. This indicates the difficulty of performing anastomosis in laparoscopic surgery.

In endoscopic surgery, the method of performing a handsewn gastroduodenostomy by making a small incision in the upper abdomen after a distal gastrectomy is commonly performed [1, 2]. However, in recent years, anastomosis using automatic suturing instruments has been mainly used for Billroth-I intracorporeal anastomosis, including a square suturing method[13] developed by Tanimura et al. [14] a hand-assisted method [14-16], an overlap method[4] developed by Uyama et al. [3] and $\delta$ anastomosis reported by Kanaya et al. [2].The disadvantages of mechanical anastomosis are characterized by the fact that it is affected by the degrees of freedom of the duodenum and that many automatic suturing instruments (namely 3-6) are used. However, the anastomotic opening is large, the procedure is simple, and the duration of anastomosis is short. 
Since Taniguchi et al. [15] reported a case of intraperitoneal anastomosis under a total laparoscopic approach, there have been no reports of laparoscopic handsewn anastomosis [1, 14]. Handsewn gastroduodenal anastomosis has been avoided due to the complicated nature of the procedure. However, in recent years, high-definition laparoscopes have become widespread, and the enlarged views obtained with these laparoscopes have reduced the stress experienced by operators. Furthermore, the field of vision in the tangential direction can be improved by pulling the lesser curvature of the stomach toward the abdominal wall with anchor suture threads, and handsewn gastroduodenal anastomosis can be performed safely by adjusting the position of the port. Furthermore, handsewn sutures were not affected by the degree of freedom of the duodenum, and during anastomosis, due to the pneumoperitoneum pressure, there was almost no leakage of digestive fluid into the abdominal cavity. The postoperative endoscopy and the findings of fluoroscopy performed using a water-soluble contrast agent revealed that, because suturing instruments based on staplers were not used, the anastomosis area was soft and highly flexible, and it was believed that there was little stenosis of the anastomosis area after surgery. Although there are reports that laparoscopic surgery requires a shorter duration of hospitalization and is more economical and superior to abdominal surgery [15], because many instruments are used for anastomosis, it is not always economically superior. However, although handsewn anastomosis involves a high level of technical difficulty and many problems, including the need to perform knot-tying procedures, such as extracorporeal knottying and intracorporeal knot-tying, the stress experienced by operators can be reduced by using a high-definition laparoscope. Moreover, there are many advantages to handsewn anastomosis, including the absence of use of instruments for anastomosis and the safety of the procedure. Furthermore, cosmetically, a small $3 \mathrm{~cm}$ incision in the umbilicus is sufficient for extracting the stomach. We have treated 18 cases at our hospital, and although time was required to perform handsewn gastroduodenal anastomosis in the early cases, it is believed that the surgery time can be reduced if the procedures for anastomosis are standardized [16-18]. We plan to study and report further cases in the future.

\section{Conclusions}

Anastomosis for a laparoscopic distal gastrectomy was intracorporeally performed with no use of automatic suturing instruments. By making adjustments to these procedures, anastomosis can be performed at a level substantially similar to conventional abdominal surgery. Moreover, although we have only treated 18 cases, it is believed that handsewn anastomosis is a useful option for laparoscopic Billroth-I reconstruction considering both cost and safety.

Disclosure Katsuichi Matsuo, Hideo Shimura, Shinnosuke Tanaka, Masahiko Nakano, Tatsuya Hashimoto, Daibou Kojima, Yuichi Yamashita have no conflicts of interest or financial ties to disclose.

Open Access This article is distributed under the terms of the Creative Commons Attribution License which permits any use, distribution, and reproduction in any medium, provided the original author(s) and the source are credited.

\section{References}

1. Kitano S, Adachi Y, Shiraishi N, Suematsu T, Bando T (1999) Laparoscopic-assisted proximal gastrectomy for early gastric carcinomas. Surg Today 29:389-391

2. Kanaya S, Gomi T, Momoi H, Tamaki N, Isobe H, Katayama T, Wada Y, Ohtoshi M (2002) Delta-shaped anastomosis in totally laparoscopic Billroth I gastrectomy: new technique of intraabdominal gastroduodenostomy. J Am Coll Surg Aug 195(2): 284-287

3. Uyama I, Sugiola A, Fujita J, Komori Y, Matsui H, Soga R, Wakayama A, Okamoto K, Ohyama A, Hasumi A (1999) Completely laparoscopic extraperigastric lymph node dissection for gastric malignancies located in the middle or lower third of the stomach. Gastric Cancer 2:186-190

4. Takaori K, Nonura E, Mabuchi H, Lee SW, Agui T, Miyamoto Y, Iwamoto M, Watanabe H, Tanigawa N (2005) A secure technique of intracorporeal Roux-Y reconstruction after laparoscopic distal gastrectomy. Am J Surg 189:178-183

5. Cuschieri A, Fayers P, Fielding J, Craven J, Bancewicz J, Joypaul V, Cook P (1996) Postoperative morbidity and mortality after D1 and D2 resections for gastric cancer: preliminary results of the MRC randomized controlled surgical trial. The surgical cooperative group. Lancet 347:995-999

6. Takiguchi S, Sekimoto M, Miyake Y, Fujiwara Y, Yasuda T, Tamura S, Yano M, Taniguchi E, Ohashi S, Monden M (2003) Totally laparoscopic distal gastrectomy using the hand-sewn Billroth-I anastomosis technique: report of a case. Surg Today 33:371-374

7. Kitano S, Iso Y, Moriyama M, Sugimachi K (1994) Laparoscopyassisted Billroth I gastrectomy. Surg Laparosc Endosc 4:146-148

8. Dent DM, Madden MV, Price SK (1988) Randomized comparison of R1 and R2 gastrectomy for gastric carcinoma. Br J Surg 75:110-111

9. Ohgami M, Otani Y, Kumai K, Kubota T, Kim YI, Kitajima M (1999) Curative laparoscopic surgery for early gastric cancer: five years experience. World J Surg 23:187-193

10. Shimizu S, Uchiyama A, Mizumoto K, Morisaki T, Nakamura K, Shimura H, Tanaka M (2000) Laparoscopically assisted distal gastrectomy for early gastric cancer: is it superior to open surgery? Surg Endosc 14:27-31

11. Adachi Y, Shiraishi N, Shiromizu A, Bavdoh T, Aramaki M, Kitano S (2000) Laparoscopy-assisted Billroth I gastrectomy compared with conventional open gastrectomy. Arch Surg 135:806-810

12. Asao T, Hosouchi Y, Nakabayashi T, Haga N, Mochiki E, Kuwano H (2001) Laparoscopically assisted total or distal gastrectomy with lymph node dissection for early gastric cancer. Br J Surg 88:128-132 
13. Reyes CD, Weber KJ, Gagner M, Divino CM (2001) Laparoscopic versus open gastrectomy: a retrospective review. Surg Endosc 15:928-931

14. Tanimura S, Higashino M, Fukunaga Y, Osugi H (2008) Intracorporeal Billroth-I reconstruction by triangulating stapling technique after laparoscopic distal gastrectomy for gastric cancer. Surg Laparosc Endosc Percutan Tech 18(1):54-58

15. Taniguchi S, Koga K, Ibusuki K, Sugio K, Uchimura Y (1997) Laparoscopic pylorus-preserving gastrectomy with intracorporeal hand-sewn anastomosis. Surg Laparosc Endosc 7:354-356

16. Adachi Y, Shiraishi N, Ikebe K, Aramaki M, Bandoh T, Kitano S (2001) Evaluation of the cost for laparoscopic-assisted Billroth I gastrectomy. Surg Endosc 15:932-936
17. Yasuda K, Shiraishi N, Inomata M, Fujii K, Sonoda K, Kitano S (2003) Learning curve for laparoscopy-assisted distal gastrectomy. Dig Endosc 15:280-283

18. Kunisaki C, Makino H, Yamamoto N, Sato T, Oshima T, Nagano Y, Fujii S, Akiyama H, Otsuka Y, Ono HA, Kosaka T, Takagawa R, Shimada H (2008) Learning curve for laparoscopy-assisted distal gastrectomy with regional lymph node dissection for early gastric cancer. Surg Laparosc Endosc Percutan Tech 18(3):236-241 